\title{
Pruritus and insomnia in hemodialysis patients; association with SF36 quality of life and clinical outcomes
}

\author{
Tayebeh Soleymanian $^{1 *(0)}$, Saba Alidadiani ${ }^{2}$, Akram Mahdavi ${ }^{1}$ \\ ${ }^{1}$ Nephrology Research Center, Tehran University of Medical Sciences, Tehran, Iran \\ ${ }^{2}$ International Branch, Nephrology Division of Shariati Hospital, Tehran University of Medical Sciences, Tehran, Iran
}

\section{A R T I C L E I N F 0}

Article Type:

Original

Article History:

Received: 10 October 2017

Accepted: 10 January 2018

Published online: 5 February 2018

\section{Keywords:}

Hemodialysis

Pruritus

Insomnia

Clinical outcomes

\begin{abstract}
A B S T R A C T
Introduction: Pruritus involves many hemodialysis (HD) patients and has a close association with sleep quality and health-related quality of life.

Objectives: The aim of this study was to determine predictors of pruritus and insomnia, the effect of pruritus on the severity of sleeping difficulties, and also their impacts on the health quality, hospitalization and mortality in a multicenter cohort of HD patients.

Patients and Methods: Pruritus and sleep problems data were obtained in $416 \mathrm{HD}$ patients from nine dialysis facilities in September 2012. SF36 quality of life and a comprehensive questionnaire was provided for the patients. Patients were followed for a median of 28 months. Unadjusted and adjusted odds ratio (AOR) of having pruritus/sleep disturbances for different variables and relative risk of death was calculated.

Results. Moderate to extreme pruritus and insomnia were respectively identified in $38 \%$ and $49 \%$ of HD patients. Independent predictors of having severe to extreme pruritus were hyperphosphatemia (mg/dL) (AOR: 1.34; 95\% CI: 1.06-1.70), anemia (g/dL) (AOR: 1.27; 95\% CI: 1.04-1.56), and older age (year) (AOR: 1.02; 95\% CI: 1.00-1.04). These predictors for severe to extreme insomnia were dialysis vintage (year) (AOR: 1.14; 95\% CI: 1.04-1.26), worse bodily pain (AOR: 1.01; 95\% CI: 1.00-1.03), poor mental health (AOR: 1.02; 95\% CI: 1.01-1.04), and severe to extreme pruritus (AOR: 8.80; 95\% CI: 3.24-23.91). SF36 quality of life was becoming significantly lower with increasing the degree of pruritus/insomnia. Likewise, hospitalization was more common in these patients. During the follow up $123(29.6 \%)$ patients passed away. The full-adjusted relative risk of death for extreme insomnia was 1.72 (95\% CI: 1.00-3.03; $P=0.05)$.

Conclusion: Moderate to extreme pruritus/insomnia are quite common in HD patients and significantly affect quality of life. Pruritus is a great predictor of insomnia. Extreme insomnia is an independent predictor of death.
\end{abstract}

Implication for health policy/practice/research/medical education:

Moderate to extreme pruritus/insomnia are quite common in HD patients and significantly affect quality of life. Pruritus is a great predictor of insomnia. Extreme insomnia is an independent predictor of death.

Please cite this paper as: Soleymanian T, Alidadiani S, Mahdavi A. Pruritus and insomnia in hemodialysis patients; association with SF36 quality of life and clinical outcomes. J Renal Inj Prev. 2018;7(4):253-258. DOI: 10.15171/jrip.2018.57.

\section{Introduction}

Pruritus is a common distressing symptom in HD patients, which has a negative impact on their quality of life (1). Factors like underlying comorbidities and inflammation, anemia, increased levels of serum phosphorus and parathyroid hormone (PTH), and skin dryness have been introduced as the main offenders of uremic pruritus (2-6). Pathogenesis of pruritus is complex and engages resident cells (keratinocytes, mast cells and sensory neurons) and temporary inflammatory cells ( $\mathrm{T}$ lymphocytes and eosinophils) (6-9). Treatment of chronic uremic pruritus is terribly sophisticated and usually unsuccessful (10-15). 
Insights into the predisposing manageable factors can help physician for better handling of this problem.

Most of the recent studies have been reported uremic pruritus in $22 \%$ to $57 \%$ of patients on hemodialysis (HD) $(2,16)$. Pruritus has a negative impact on health-related quality of life and also causes sleeping difficulties in dialysis patients (17). Insomnia is common in chronically ill patients and it largely depends on the severity of underlying comorbid conditions. Uremic patients on dialysis specifically complain about insomnia and other sleeping problems $(18,19)$. It has been proposed that sleep disturbances would raise cardiovascular events and mortality in general population and patients with endstage renal disease (ESRD) (20,21).

\section{Objectives}

The aim of this study was to determine predictors of pruritus and insomnia, the effect of pruritus on the severity of sleeping difficulties, and also their impacts on the health quality, hospitalization and mortality in a multicenter cohort of HD patients.

\section{Patients and Methods}

\section{Study population and research design}

In this observational study, 532 maintenance HD patients from nine facilities were enrolled in September 2012. The enrolled facilities which their authorities and medical staff signed to collaborate were from three different regions. Two facilities with 167 patients from north of Tehran, five facilities with 217 participants from center of Tehran and two facilities with 148 patients from south of Tehran. All patients had to be at least 18 years old while receiving outpatient HD at least for 2 weeks. Out of 532 patients, 416 subjects (95\% on dialysis for more than three months) agreed to complete the SF36 questionnaire (validated Persian translation) which also comprised questions about kidney disease specific symptoms including pruritus and sleeping problems. The SF36 comprises 36 questions which 35 of them are used to make 8 multi-item subscales, which are 1) physical functioning, 2) role-physical, 3) bodily pain, 4) general health, 5) vitality, 6) social functioning, 7) role-emotional, and 8) mental health (8). A score from 0 (lowest HRQOL) to 100 (highest HRQOL) is obtained for each. These subscales of SF36 are condensed into two components. The first five subscales constitute of physical component summary (PCS) and the last five make up mental component summary (MCS), hence the subscales of general health and vitality are used in both components. The scores of the total SF36 score and the two components are counted as averaging of the subscales. The Cronbach's a coefficient for each subscale was $>0.88$. We also asked about the severity of itching and sleeping problems of our patients in the provided questionnaire. The two included inquiries were "during the past 4 weeks how much have you been bothered by itching/difficulty sleeping?" with five-scale answers of "not at all, mild, moderate, severe, extremely".
In addition, a comprehensive questionnaire comprising all demographics, and laboratory data was filled out by using administrative data and medical records for all patients. At least two to three constitutive laboratory data at the study entry were recorded in the questionnaire (their mean was used for analysis). Then, patients were followed up until February 2015. During follow up period (median of 28 months; minimum of 0.5 and maximum of 30 months) causes of hospitalizations and exit from HD including death was recorded. The last follow up time was the last visit or whenever patients left HD because of renal recovery, transfer to peritoneal dialysis (PD) or undergoing transplantation (one month after PD transfer or transplantation). Patients who transferred to a different facility were followed there. The study was approved by the specialized review boards and ethics committees. Informed patient consent was obtained.

\section{Ethical issues}

1) The research followed the tenets of the Declaration of Helsinki and its later amendments; 2) informed consent was obtained; and 3) permission of the ethical review committee of Tehran University of Medical Sciences was obtained prior to execution of the study.

\section{Statistical methods}

Demographic characteristics and laboratory data of the patients are summarized using percentage of the total and means ( \pm standard deviation; SD) or medians (interquartile range $[\mathrm{IQR}])$ as appropriate. Mean values of two or three laboratory results at study start for each patient were used in the analyses. Categorical variables were compared using Fisher's exact test, and continuous variables were compared using ANOVA and Kruskal-Wallis tests as appropriate. Logistic regression analysis was used to estimate the odds ratio (OR) of different variables predicting the severeextreme pruritus/insomnia versus negative pruritus/ insomnia after adjusting for case-mix covariates (age, sex, dialysis vintage, and body mass index [BMI]), diabetes, and significant laboratory data in univariate analysis. We also estimated unadjusted and incremental adjusted (for case-mix, serum albumin, and diabetes) relative risk (RR) of death using Logistic regression analysis for patients with severe-extreme pruritus/insomnia. Patient survival for patients with severe-extreme pruritus/insomnia versus those with no pruritus/insomnia was estimated by the Kaplan-Meier method. Multivariate regression analysis was used for detecting the most significant predictors of SF36 score. The data analysis was performed using SPSS version 19 (SPSS Inc., Chicago, IL). Significant level was considered as $P<0.05$.

\section{Results}

The mean age of the patients was $57.2 \pm 15.4$ years (range: $18-93$ years) which $56.5 \%(n=235)$ of them were men and $42 \%(n=174)$ were diabetic. Other causes of ESRD were glomerular diseases $(n=63,15 \%)$; urologic 
problems $(n=42,10 \%)$; hereditary disorders $(n=21$, $5 \%)$; and hypertension, unknown and other uncommon causes $(\mathrm{n}=116,28 \%)$. The mean duration on dialysis was $45.6 \pm 47.8($ median $=27$, interquartile range $=11-68)$ months. The mean \pm SD for BMI was $24.5 \pm 4.5 \mathrm{~kg} / \mathrm{m}^{2}$, serum albumin; $3.91 \pm 0.36 \mathrm{~g} / \mathrm{dL}$, hemoglobin; $10.6 \pm 1.5$ g/dL, phosphorus: $5.41 \pm 1.26 \mathrm{mg} / \mathrm{dL}$, calcium; $8.86 \pm 0.71$ mg/dL, PTH: 341 (IQR: 165-586) pg/mL, Kt/V; $1.31 \pm 0.19$, Short Form 36 (SF36); $50 \pm 21$, PCS; $51 \pm 22$ and MCS; $53 \pm 22$. The median of annual admission frequency was 0.80 (IQR: $0.00-1.55)$. During follow up period (747 patient years), 123 patients died (29.6\%). Causes of death included cardiovascular disease (CVD) (37\%), other causes superimposed on CVD (11.5\%), infections superimposed on CVD (20.5\%), infections (4\%), malignancy (7.5\%), cachexia $(5.5 \%)$, and other causes $(14 \%)$.

Thirty-eight percent of our patients reported having moderate to extreme pruritus. The proportion of severe to extreme pruritus was $18 \%$. In univariate analysis, there was a significant difference in serum hemoglobin, phosphorus, SF36 score and its components, annual admission frequency, and death among patients with various degree of pruritus (Table 1). Independent predictors of having severe to extreme pruritus (reference; no pruritus) were hyperphosphatemia (mg/dL) (adjusted odds ratio or AOR: 1.34; 95\% CI: $1.06-1.70 ; P=0.01)$, anemia (g/dL) (AOR; 1.27; 95\% CI: 1.04-1.56; $P=0.02$ ), and older age (year) (AOR: 1.02; 95\% CI: 1.00-1.04; $P=0.05)$.

Forty-nine percent of our patients complained about moderate to extreme insomnia. The proportion of severe to extreme insomnia was $21 \%$. In univariate analysis, there was a significant difference in dialysis vintage, bodily pain (subscale of SF36 score), mental health (subscale of SF36 score), SF36 score and its components, severe to extreme pruritus, and death among patients with various degree of insomnia (Table 2). Independent predictors of having severe to extreme insomnia (reference; no insomnia) were dialysis vintage (year) (AOR: 1.14; 95\% CI: 1.04-1.26; $P=0.01$ ), worse bodily pain (AOR: 1.01 ; 95\% CI: 1.00 $1.03 ; P=0.05$ ), poor mental health (AOR: 1.02 ; 95\% CI: $1.01-1.04 ; P=0.02$ ), and severe to extreme pruritus (AOR: 8.80; 95\% CI: 3.24-23.91; $P<0.001)$ ). It is noteworthy to say that higher scores for SF36 and its components or subscales indicate a better quality of life, therefore lower scores of bodily pain and mental health subscales mean higher pain and worse mental health.

Unadjusted relative risk of death for severe-extreme pruritus (reference: no pruritus) was 1.38 (95\% CI: 1.012.45; $P=0.05$ ), case-mix adjusted RR was 1.29 (95\% CI: $0.98-2.30 ; P=0.09$ ), and fully adjusted (case-mix, albumin, and diabetes) RR was 1.00 (95\% CI: 0.49-1.72; $P=0.39$ ). These numbers for severe-extreme insomnia (reference: no insomnia) were respectively 1.71 (95\% CI: 1.01-3.08; $P=0.04), 1.87$ (95\% CI: $1.02-3.55 ; P=0.03), 1.12$ (0.56$2.71 ; P=0.49)$. However, for extreme insomnia the full adjusted relative risk of death was 1.72 (95\% CI: 1.00-3.03; $P=0.05)$. Kaplan-Meier survival of patients with severeextreme pruritus/insomnia is shown in Figure 1 (A, B). In full adjusted model, other predictors of mortality were age (year) 1.04 (95\% CI: 1.02-1.06; $P=0.001)$, serum albumin (g/dL) 4.29 (95\% CI: 1.89-9.80; $P=0.001)$, and

Table 1. Comparison of different variables in hemodialysis patients with various degrees of pruritus

\begin{tabular}{|c|c|c|c|c|c|c|}
\hline Characteristic & $\begin{array}{c}\text { Not } \\
(n=157 ; 38 \%)\end{array}$ & $\begin{array}{c}\text { Mild } \\
(n=100 ; 24 \%)\end{array}$ & $\begin{array}{c}\text { Moderate } \\
(n=84 ; 20 \%)\end{array}$ & $\begin{array}{c}\text { Severe } \\
(n=36 ; 8.5 \%)\end{array}$ & $\begin{array}{c}\text { Extreme } \\
(n=39 ; 9.5 \%)\end{array}$ & $P$ value \\
\hline \multicolumn{7}{|l|}{ Demographic data } \\
\hline Age (y) & $56.1 \pm 15.9$ & $57.7 \pm 13.5$ & $58.3 \pm 16.8$ & $56.6 \pm 16.4$ & $58.7 \pm 14.2$ & 0.54 \\
\hline Sex (female \%) & $44 \%$ & $38 \%$ & $45 \%$ & $42 \%$ & $53 \%$ & 0.60 \\
\hline Dialysis vintage $(y)^{*}$ & $2.33(0.83-5.79)$ & $2.25(1.00-5.45)$ & $2.16(1.00-5.41)$ & $1.79(0.60-5.58)$ & $3.00(0.84-6.85)$ & 0.76 \\
\hline Diabetes (yes \%) & $40 \%$ & $43 \%$ & $41 \%$ & $39 \%$ & $50 \%$ & 0.63 \\
\hline Body mass index $\left(\mathrm{kg} / \mathrm{m}^{2}\right)$ & $23.9 \pm 3.8$ & $24.8 \pm 4.2$ & $24.9 \pm 4.6$ & $24.6 \pm 5.1$ & $24.7 \pm 6.3$ & 0.41 \\
\hline \multicolumn{7}{|l|}{ Laboratory data (serum) } \\
\hline Albumin (g/dL) & $3.93 \pm 0.37$ & $3.87 \pm 0.32$ & $3.95 \pm 0.37$ & $3.89 \pm 0.44$ & $3.79 \pm 0.30$ & 0.16 \\
\hline Hemoglobin (g/dL) & $10.6 \pm 1.6$ & $10.7 \pm 1.6$ & $10.7 \pm 1.5$ & $10.3 \pm 1.6$ & $9.8 \pm 1.4$ & 0.03 \\
\hline Creatinine (mg/dL) & $8.8 \pm 2.7$ & $8.7 \pm 2.7$ & $8.3 \pm 2.6$ & $8.6 \pm 2.8$ & $8.2 \pm 2.9$ & 0.54 \\
\hline Single pool Kt/V & $1.33 \pm 0.19$ & $1.31 \pm 0.19$ & $1.28 \pm 0.18$ & $1.28 \pm 0.23$ & $1.31 \pm 0.21$ & 0.46 \\
\hline Calcium (mg/dL) & $8.83 \pm 0.73$ & $8.95 \pm 0.73$ & $8.88 \pm 0.65$ & $8.83 \pm 0.64$ & $8.68 \pm 0.76$ & 0.37 \\
\hline Phosphorus (mg/dL) & $5.3 \pm 1.2$ & $5.2 \pm 1.2$ & $5.3 \pm 1.2$ & $5.4 \pm 1.3$ & $6.2 \pm 1.6$ & 0.004 \\
\hline Intact PTH (pg/ml)* & $298(157-554)$ & $325(143-503)$ & $284(156-682)$ & $419(252-636)$ & $420(274-777)$ & 0.06 \\
\hline Ferritin (ng/ml) & $424 \pm 257$ & $392 \pm 259$ & $459 \pm 273$ & $397 \pm 324$ & $522 \pm 372$ & 0.14 \\
\hline SF36 score & $56 \pm 21$ & $51 \pm 20$ & $45 \pm 18$ & $43 \pm 21$ & $35 \pm 18$ & $<0.001$ \\
\hline Physical component summary & $58 \pm 21$ & $52 \pm 20$ & $46 \pm 18$ & $43 \pm 23$ & $36 \pm 19$ & $<0.001$ \\
\hline Mental component summary & $59 \pm 21$ & $54 \pm 21$ & $49 \pm 20$ & $45 \pm 21$ & $39 \pm 21$ & $<0.001$ \\
\hline \multicolumn{7}{|l|}{ Clinical outcomes } \\
\hline Hospitalization (per patient-year) * & $0.44(0.00-1.50)$ & $0.62(0.00-1.47)$ & $0.43(0.00-1.32)$ & $0.80(0.00-1.71)$ & $1.45(0.41-4.00)$ & 0.02 \\
\hline Death (yes \%) & $32 \%$ & $23 \%$ & $25 \%$ & $25 \%$ & $53 \%$ & 0.01 \\
\hline
\end{tabular}


Table 2. Comparison of different variables in hemodialysis patients with various degrees of Insomnia

\begin{tabular}{|c|c|c|c|c|c|c|}
\hline Characteristic & $\begin{array}{c}\text { Not } \\
(n=132 ; 32 \%)\end{array}$ & $\begin{array}{c}\text { Mild } \\
(n=81 ; 19 \%)\end{array}$ & $\begin{array}{c}\text { Moderate } \\
(n=115 ; 28 \%)\end{array}$ & $\begin{array}{c}\text { Severe } \\
(n=42 ; 10 \%)\end{array}$ & $\begin{array}{c}\text { Extreme } \\
(n=46 ; 11 \%)\end{array}$ & $P$ value \\
\hline \multicolumn{7}{|l|}{ Demographic data } \\
\hline Age (y) & $57.1 \pm 16.3$ & $56.8 \pm 16.1$ & $57.5 \pm 13.6$ & $53.9 \pm 15.8$ & $59.5 \pm 15.4$ & 0.54 \\
\hline Sex (female \%) & $36 \%$ & $50 \%$ & $40 \%$ & $52 \%$ & $52 \%$ & 0.11 \\
\hline Dialysis vintage $(y)^{*}$ & $1.91(0.83-5.14)$ & $1.75(0.63-5.25)$ & $2.33(0.91-5.29)$ & $3.29(1.16-7.22)$ & 3.95 (1.39-7.79) & 0.04 \\
\hline Diabetes (yes \%) & $40 \%$ & $42 \%$ & $42 \%$ & $41 \%$ & $46 \%$ & 0.87 \\
\hline Body mass index $\left(\mathrm{kg} / \mathrm{m}^{2}\right)$ & $24.2 \pm 4.1$ & $24.6 \pm 4.4$ & $24.3 \pm 3.7$ & $24.2 \pm 4.2$ & $25.3 \pm 6.9$ & 0.63 \\
\hline \multicolumn{7}{|l|}{ Laboratory data (serum) } \\
\hline Albumin (g/dL) & $3.92 \pm 0.35$ & $3.90 \pm 0.40$ & $3.93 \pm 0.34$ & $3.89 \pm 0.38$ & $3.81 \pm 0.32$ & 0.39 \\
\hline Hemoglobin (g/dL) & $10.6 \pm 1.5$ & $10.5 \pm 1.6$ & $10.6 \pm 1.5$ & $10.4 \pm 1.4$ & $10.3 \pm 1.6$ & 0.67 \\
\hline Creatinine (mg/dL) & $8.6 \pm 2.6$ & $8.5 \pm 2.6$ & $8.7 \pm 2.9$ & $9.1 \pm 2.8$ & $8.3 \pm 2.7$ & 0.65 \\
\hline Single pool Kt/V & $1.32 \pm 0.21$ & $1.29 \pm 0.19$ & $1.29 \pm 0.18$ & $1.35 \pm 0.22$ & $1.32 \pm 0.17$ & 0.50 \\
\hline Calcium (mg/dL) & $8.85 \pm 0.73$ & $8.78 \pm 0.63$ & $8.95 \pm 0.72$ & $8.81 \pm 0.67$ & $8.76 \pm 0.74$ & 0.42 \\
\hline Phosphorus (mg/dL) & $5.24 \pm 1.18$ & $5.34 \pm 1.21$ & $5.52 \pm 1.25$ & $5.49 \pm 1.38$ & $5.70 \pm 1.49$ & 0.18 \\
\hline Intact PTH (pg/ml)* & $316(209-520)$ & $339(166-548)$ & $374(159-624)$ & $364(204-736)$ & $360(139-797)$ & 0.78 \\
\hline Ferritin (ng/ml) & $436 \pm 258$ & $386 \pm 268$ & $442 \pm 271$ & $447 \pm 322$ & $452 \pm 335$ & 0.63 \\
\hline Pruritus (severe-extreme) & $14 \%$ & $21 \%$ & $39 \%$ & $58 \%$ & $66 \%$ & $<0.001$ \\
\hline SF36 Score & $58 \pm 21$ & $51 \pm 20$ & $49 \pm 18$ & $42 \pm 19$ & $32 \pm 15$ & $<0.001$ \\
\hline Physical component summary & $60 \pm 21$ & $52 \pm 21$ & $50 \pm 18$ & $42 \pm 21$ & $31 \pm 17$ & $<0.001$ \\
\hline Mental component summary & $61 \pm 22$ & $54 \pm 21$ & $51 \pm 18$ & $46 \pm 21$ & $34 \pm 17$ & $<0.001$ \\
\hline Bodily pain subscale & $73 \pm 26$ & $64 \pm 29$ & $59 \pm 30$ & $54 \pm 34$ & $31 \pm 28$ & $<0.001$ \\
\hline Mental health subscale & $68 \pm 26$ & $60 \pm 23$ & $57 \pm 23$ & $54 \pm 30$ & $40 \pm 24$ & $<0.001$ \\
\hline \multicolumn{7}{|l|}{ Clinical outcomes } \\
\hline Hospitalization (per patient-year)* & $0.43(0.00-1.48)$ & $0.84(0.00-1.81)$ & $0.50(0.00-1.33)$ & $0.80(0.30-1.52)$ & $1.38(0.00-3.03)$ & 0.08 \\
\hline Death (yes \%) & $25 \%$ & $32 \%$ & $24 \%$ & $27 \%$ & $48 \%$ & 0.04 \\
\hline
\end{tabular}

*Results are shown by median and interquarter range.

diabetes $1.85(1.06-3.24 ; P=0.03)$.

In multivariate regression analysis, after mixing of covariates (including patient characteristics, laboratory data and comorbidities) which were significant in univariate analysis for SF36 score; $20 \%$ variation of SF36 score was respectively explained by insomnia and pruritus.

\section{Discussion}

This study provides a description of self-reported uremic pruritus and insomnia in $416 \mathrm{HD}$ patients from nine HD facilities with a detail of laboratory data, SF36 measure, hospitalization, and mortality. We evaluated the prevalence of pruritus and insomnia, their risk factors, association with SF36 scores and its components, relationship between pruritus and severity of insomnia, the most important factors that determine quality of life and the impact of pruritus and insomnia on hospitalization rate and mortality.

Moderate to extreme pruritus was seen in $38 \%$ of our patients which is in the range of recent reports $(2,16)$. For determining the associations of pruritus, we compared patients with severe to extreme pruritus with those who reported having pruritus not at all (reference group) after adjustment for case-mix, diabetes and different laboratory data. We found that anemia, hyperphosphatemia, and older age were the most significant independent risk factors for having severe to extreme pruritus which was in agreement with most other reports (2-6). Indeed, adjusted Odds Ratio of having severe-extreme pruritus for patients with hemoglobin $<10 \mathrm{~g} / \mathrm{dL}$ was $2.25(95 \%$
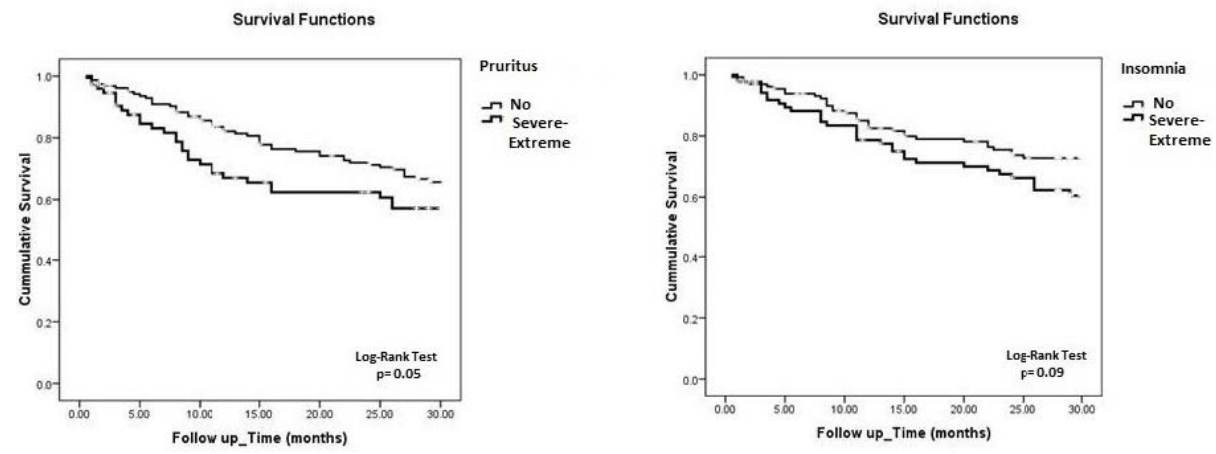

Figure 1. Kaplan-Meier survival for patients with severe-extreme pruritus (A)/insomnia (B) versus patients with negative pruritus (A)/insomnia (B). 
CI: 1.01-4.85), for patients with serum phosphorus $\geq 6$ $\mathrm{mg} / \mathrm{dL}$ was 1.66 (95\% CI: 1.01-3.15), and for patients older than 65 years was 1.55 (95\% CI: 1.00-3.05). There was a strong, inverse correlation $(\mathrm{r}=-0.31, P<0.001)$ between severity of pruritus and SF36 scores; and also physical and mental summary components $(r=-0.33$ and $P=-0.29$, respectively). Unlike some studies $(2,22)$, we did not observe an association between $\mathrm{Kt} / \mathrm{V}$ and severity of pruritus.

Our findings mostly are in line with other reports which have shown hyperphosphatemia and higher serum calcium $\times$ phosphorus motivate pruritus, and may be related to its local effect on skin $(2,23)$. Both immune and opioid hypothesis have been proposed as mechanisms for uremic pruritus (2-6). It was shown that $\mathrm{T}$ helper cells, C-reactive protein and interleukin-6 levels are higher in HD patients with pruritus $(8,9)$. Higher pruritus in older patients in our study possibly is associated with more skin dryness caused by sweat gland atrophy in these patients. In the present study, the prevalence of moderate to extreme insomnia was $49 \%$ and the most significant predictor of insomnia was severe-extreme pruritus with AOR of 8.80 (95\% CI: 3.24-23.91). Moderate to extreme insomnia was recognized in $73 \%$ of patients with severe-extreme pruritus. There was strong $(P<0.001)$ inverse correlation between insomnia and SF36 score $(r=-0.36)$, and PCS $(\mathrm{r}=-0.37)$ and MCS $(\mathrm{r}=-0.34)$. It was demonstrated that uremic pruritus is aggravated at night and could affect sleep quality and also quality of life of the HD patients $(16,17)$. In addition, disruption of sleep in severe pruritus results in remarkable weakness and depression $(2,16)$. We also noticed that insomnia was significantly more common in patients with longer duration on dialysis and in those with worse bodily pain and poor mental health. Our findings mostly are in agreement with other studies $(24,25)$.

"Severe-extreme" pruritus/insomnia was associated with higher mortality and although after adjustment they were no longer predictors of higher death, still patients with "extreme" insomnia had 2.5 folds higher adjusted mortality. Some studies have shown that insomnia leads to higher death among ESRD patients by increasing systemic inflammation and accompanied cardiovascular disease $(20,21)$. Moreover, one report indicated that severe pruritus may be an independent risk for mortality (2). Both pruritus and insomnia had a negative impact on physical component summary and mental component summary of SF36 measure. Actually, in multivariate regression analysis, 20\% variation in SF36 score was explained by the presence of insomnia and pruritus. Therefore, applying measures to alleviate these two important precipitating factors and prescribing medications which mitigate these symptoms can largely promote quality of life of HD patients.

In this study hospitalization rate was higher in patients with severe-extreme pruritus/insomnia, however adjusted hospitalization rate did not differ with other groups which emphasized the poor underlying conditions of these patients.

\section{Conclusion}

In summary, the prevalence of moderate to severe pruritus and insomnia in HD patients were 38\% and 49\%, respectively. Hyperphosphatemia, anemia, and older age were risk factors for severe-extreme pruritus. The most important predictor of severe-extreme insomnia was pruritus. Both severe-extreme pruritus and insomnia were associated with poor quality of life. In addition, extreme insomnia was an independent predictor of mortality.

\section{Limitations of the study}

SF36 score, pruritus and insomnia in this study were evaluated only once. All of them (and also laboratory data) would be changed along the time course. However, for laboratory data we considered the mean of three constitutive results at the study start. Although SF36 score and insomnia had some impact on clinical outcome, still the causal effect of them on outcome should further be determined by RCTs.

Acknowledgments

Special thanks to the staff of the nine participating HD centers.

\section{Authors' contribution}

TS conducted the research. SA and AM collected the data. TS analyzed the data. TS prepared the primary and final draft. All authors signed the manuscript.

\section{Conflicts of interest}

The authors declare no conflict of interest.

\section{Ethical considerations}

Ethical issues (including plagiarism, data fabrication, double publication) have been completely observed by the authors.

\section{Funding/Support}

This study was funded by Tehran and Shahid Beheshti University of Medical Sciences, Tehran, Iran (Grant No. 910825-12).

\section{References}

1. Curtin RB, Bultman DC, Thomas-Hawkins C, Walters BAJ, Schatell D. Hemodialysis patients' symptom experiences: effects on physical and mental functioning. Nephrol Nursing J. 2002; 29: 562-74.

2. Narita I, Alchi B, Omori K, Sato F, Ajiro J, Saga D, et al. Etiology and prognostic significance of severe uremic pruritus in chronic hemodialysis patients. Kidney Int. 2006; 69:1626-32.

3. Massry SG, Popovtzer MM, Coburn JW, Makoff DL, 
Maxwell MH, Kleeman CR. Intractable pruritus as a manifestation of secondary hyperparathyroidism in uremia. Disappearance of itching after subtotal parathyroidectomy. N Engl J Med. 1968; 279:697-700.

4. De Marchi S, Cecchin E, Villalta D, Sepiacci G, Santini G, Bartoli E. Relief of pruritus and decreases in plasma histamine concentrations during erythropoietin therapy in patients with uremia. N Engl J Med. 1992; 326:969-74.

5. Charlesworth EN, Beltrani VS. Pruritic dermatoses: overview of etiology and therapy. Am J Med. 2002;113 Suppl 9A:25S-33S.

6. Virga G, Visentin I, La Milia V, Bonadonna A. Inflammation and pruritus in haemodialysis patients. Nephrol Dial Transplant. 2002;17:2164-9.

7. Mettang T, Pauli-Magnus C, Alscher DM. Uraemic pruritus-new perspectives and insights from recent trials. Nephrol Dial Transplant. 2002;17:1558-63.

8. Kimmel M, Alscher DM, Dunst R, Braun N, Machleidt $\mathrm{C}$, Kiefer T, et al. The role of micro-inflammation in the pathogenesis of uremic pruritus in hemodialysis patients. Nephrol Dial Transplant. 2006;21:749-55.

9. Fallahzadeh MK, Roozbeh J, Geramizadeh B, Namazi MR. Interleukin-2 serum levels are elevated in patients with uremic pruritus: a novel finding with practical implications. Nephrol Dial Transplant. 2011;26:3338-44. doi: 10.1093/ ndt/gfr053.

10. Weisshaar E, Dunker N, Gollnick H. Topical capsaicin therapy in humans with hemodialysis-related pruritus. Neurosci Lett. 2003;345:192-4.

11. Pauli-Magnus C, Mikus G, Alscher DM, Kirschner T, Nagel W, Kiefer T et al. Naltrexone does not relieve uremic pruritus: results of a randomized, double-blind, placebocontrolled crossover study. J Am Soc Nephrol. 2000;11:5149.

12. Kuypers DR, Claes K, Evenepoel P, Maes B, Vanrenterghem Y. A prospective proof of concept study of the efficacy of tacrolimus ointment on uraemic pruritus (UP) in patients on chronic dialysis therapy. Nephrol Dial Transplant. 2004;19:1895-901.

13. Gunal AI, Ozalp G, Yoldas TK, Gunal SY, Kirciman E, Celiker H. Gabapentin therapy for pruritus in haemodialysis patients: a randomized, placebo-controlled, double-blind trial. Nephrol Dial Transplant. 2004;19:3137-3139.

14. Gilchrest BA, Rowe JW, Brown RS, Steinman TI, Arndt KA.
Ultraviolet phototherapy of uremic pruritus. Long-term results and possible mechanisms of action. Ann Intern Med. 1979;91:17-21.

15. Shavit L, Grenader T, Lifschitz M, Slotki I. Use of pregabalin in the management of chronic uremic pruritus. J Pain Symptom Manage. 2013;45:776-781. doi: 10.1016/j. jpainsymman.2012.03.001.

16. Pisoni RL, Wikström B, Elder SJ, Akizawa T, Asano Y, Keen ML et al. Pruritus in haemodialysis patients: International results from the Dialysis Outcomes and Practice Patterns Study (DOPPS). Nephrol Dial Transplant. 2006; 21:34953505.

17. Yosipovitch G, Zucker I, Boner G, Gafter U, Shapira $\mathrm{Y}$, David $\mathrm{M}$, et al. A questionnaire for the assessment of pruritus: validation in uremic patients. Acta Derm Venereol. 2001;81:108-11.

18. Murtagh FE, Addington-Hall J, Higginson IJ. The prevalence of symptoms in end-stage renal disease: a systematic review. Adv Chronic Kidney Dis. 2007;14:82-99.

19. Parfrey PS, Vavasour HM, Henry S, Bullock M, Gault MH. Clinical features and severity of nonspecific symptoms in dialysis patients. Nephron. 1988;50:121-8.

20. Elder SJ, Pisoni RL, Akizawa T, Fissell R, Andreucci VE, Fukuhara S, et al. Sleep quality predicts quality of life and mortality risk in haemodialysis patients: results from the Dialysis Outcomes and Practice Patterns Study (DOPPS). Nephrol Dial Transplant. 2008;23:998-1004.

21. Chiu YL, Chuang YF, Fang KC, Liu SK, Chen HY, Yang JY et al. Higher systemic inflammation is associated with poorer sleep quality in stable haemodialysis patients. Nephrol Dial Transplant. 2009;24:247-51.

22. Hiroshige K, Kabashima N, Takasugi M, Kuroiwa A. Optimal dialysis improves uremic pruritus. Am J Kidney Dis 1995;25:413-9.

23. Blachley JD, Blankenship DM, Menter A, Parker TF 3rd, Knochel JP. Uremic pruritus: skin divalent ion content and response to ultraviolet phototherapy. Am J Kidney Dis. 1985;5:237-41.

24. Koch BC, Nagtegaal JE, Hagen EC, van Dorp WT, Boringa JB, Kerkhof GA, et al. Subjective sleep efficiency of hemodialysis patients. Clin Nephrol. 2008;70:411-6.

25. Sabbatini M, Minale B, Crispo A, Pisani A, Ragosta A, Esposito R, et al. Insomnia in maintenance haemodialysis patients. Nephrol Dial Transplant. 2002;17:852-856.

Copyright ( 2018 The Author(s); Published by Nickan Research Institute. This is an open-access article distributed under the terms of the Creative Commons Attribution License (http://creativecommons.org/licenses/by/4.0), which permits unrestricted use, distribution, and reproduction in any medium, provided the original work is properly cited. 